\title{
MINIMALLY INVASIVE PARYLENE DUAL-VALVED FLOW DRAINAGE SHUNT FOR GLAUCOMA IMPLANT
}

\author{
Jeffrey Chun-Hui Lin ${ }^{1}$, Po-Jui Chen ${ }^{1}$, Brian Yu ${ }^{1}$, Mark Humayun ${ }^{2}$, Yu-Chong Tai ${ }^{1}$ \\ ${ }^{1}$ California Institute of Technology, USA \\ ${ }^{2}$ University of Southern California, USA
}

\begin{abstract}
A parylene-enabled microvalved shunt implant for glaucoma drainage is presented in this paper. Enabled by the dual-checkvalve operation, this device can physically drain the extra intraocular fluid and regulate the intraocular pressure (IOP) within the normal range (15-20 $\mathrm{mmHg}$ ). Improved surgical features, in addition to the functional/microfluidic components, such as parylenetube carrier and anchors, are also incorporated in such device to realize minimally invasive suture-less implantation, suitable for practical in vivo use. With the optimized micromachining and post-fabrication process procedures, the developed implant is the first checkvalved glaucoma drainage device (GDD), which is passive, consumes no additional power, and functions without any circuit involved to pursue its medical application.
\end{abstract}

\section{INTRODUCTION}

It is estimated 66.8 million people worldwide have glaucoma, most of whom are associated with abnormally high intraocular pressure (IOP) $(>20 \mathrm{mmHg}$ ) [1]. The most common way to treat glaucoma is using eye drops, which either reduce the generation of aqueous humor, or help the eye drain out fluid. Usually, this kind of medication needs patients to take the eye drop regularly every morning and evening, making it inconvenient and easily forgotten. On the other hand, some patients may get refractory responses or become allergic to the eye drops. In 1907, Rollet first proposed the idea of implanting a horse-hair thread subconjuctivally trying to drain out the excess eye fluid. Subsequently, other people tried many different kinds of materials [2]. These previous hollow tubes didn't get too much success because of bio-incompatibility and migration of the implants. In addition, the hollow tube, with neither threshold pressure constraint nor high-pressure protecting mechanism, causes excess fluid to leak out of the eye, which leads to hypotony. Thus, it is desirable to have a passive device to regulate the IOP to be below $20 \mathrm{mmHg}$, along with a protecting mechanism saving the eye from unexpected high pressure (e.g. $>50 \mathrm{mmHg}$ ). This paper presents the first integrated parylene-tube-type microvalved glaucoma drainage device (GDD) incorporating microflow control system, parylene-tube carrier and anchors, which is designed to fit in a needleimplantable form factor for suture-less minimally invasive implantation through subconjunctival needle injection.

It is well known that a successful GDD must have continuous and reliable IOP control capability. In recent works, we have demonstrated that such requirements can be achieved by using dual checkvalves with flow regulation in required ranges compatible with IOP regulation specification [3]. However, their integration with appropriate surgical components for fixation is also necessary for its practical applications. After proving the concept of using the surgical features in the proposed device in MicroTAS07 [4] and demonstrating the preliminary assembly in HiltonHead08 [5], we have optimized the fabrication and packaging of a new device to create a parylene-tube GDD that targets subconjunctival implantation with needle-inserted and suture-less surgical procedures (Figure 1).

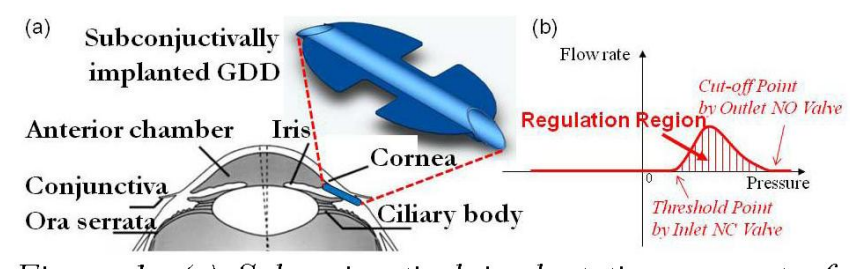

Figure 1: (a) Subconjunctival implantation concept of GDD; (b) Concept of IOP band passes regulation behavior.

\section{DESIGN}

The complete implant comprises a dual-valve microflow regulation system in a tube with integrated flexible tissue anchors (i.e., fixation). Dual back-to-back microvalves with one normally closed (NC) but open at $20 \mathrm{mmHg}$ and the other normally open (NO) but closed beyond $50 \mathrm{mmHg}$ are designed (Figure 2). A stiction prestressed NC valve is developed featuring spontaneous bonding from drying process. Few holes are designed on stiction-bonding parts where epoxy can be used to further ensure bonding strength and to prevent parylene from delamination after repeated operations. Trenches are added in the free-standing membrane of the NO valves to avoid stiction and to improve its high pressure sealing behavior.

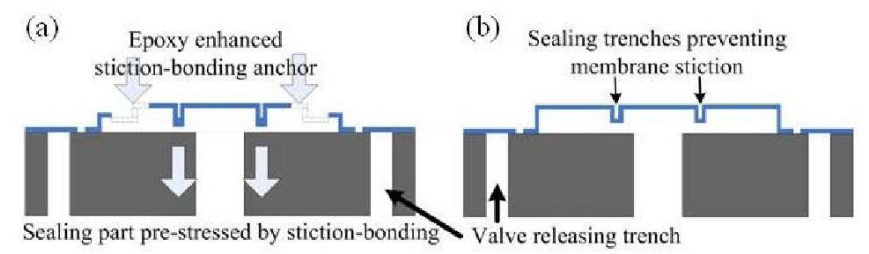

Figure 2: A dual valve micro-flow regulation system: (a) NC vavle; (b) NO valve.

To accommodate two microvalves, hollow tubes made of thick parylene are utilized and the length is chosen as $6 \mathrm{~mm}$ long (Figure 3). Both ends of these parylene tube carriers are slanted at 30 degrees to facilitate the surgical implantation and guard the GDD against iris retraction. To prevent the implanted GDD from dislodging, rollable/foldable anchors with hemispherical recesses are also designed. The anchor has a wingspan larger than the O.D. of 19-gauge hypodermic needles and can stretch out after needle retraction for robust fixation. The radius of hemispherical recesses is $300 \mu \mathrm{m}$, fitting and covering the parylene tube O.D. well during later assembly. Every parts of the GDD, 
(microvalves, parylene tube carriers, parylene anchors), is fabricated separately and finally integrated into one system. The fluid can flow from $\mathrm{NC}$ valve to $\mathrm{NO}$ valve before the pressure reaches the limit of NO valve, while the opposite direction is forbidden due to the closed NC valve.

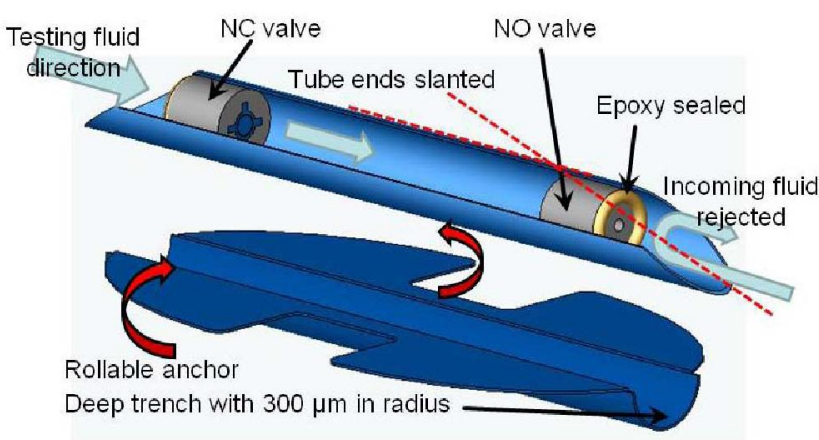

Figure 3: Full GDD system consisting of a dual-valve micro-flow regulation system, a parylene-tube carrier, and a rollableffoldable anchor.

\section{FABRICATION AND PACKAGING}

The microvalve fabrication started with backside DRIE to leave a 50- $\mu \mathrm{m}$-thick membrane (Figure 4-(a)). The circular boundary of the valve seats were also defined here with diameter to be $500 \mu \mathrm{m}$, which can be fit into parylene carrier I.D. smoothly. $\mathrm{XeF}_{2}$ was used to roughen the front side surface to improve the adhesion between parylene and the silicon valve seat. Lithography, which defines the valves' height difference was then performed and done by a 3-step exposure. After parylene coating, RIE was used to pattern coated parylene and then through holes were opened by backside DRIE. The wanted stiction parts of the circumferential of $\mathrm{NC}$ valve bonded to silicon surface simultaneously after the sacrificial photoresist were stripped and dried. Tiny epoxy drops were then applied onto these NC valves' stiction-bonding parts to make sure the parylene won't de-laminate after several repeated operations. The fabrication results of microvalves are shown in Figure 5-(a)-(c).

As for the fixation parts of the system, oxide was first patterned for later $\mathrm{XeF}_{2}$ etching (Figure 4-(b)). The wafer was isotropically etched to create semi sphere recesses with radius to be $300 \mu \mathrm{m}$ in depth. $20 \mu \mathrm{m}$ thick parylene was then coated onto the wafer. Aluminum, used as the oxygen plasma etching mask, was deposited and then patterned by dry film photolithography. Dry film is one kind of negative photoresist that is widely used in printed circuit board (PCB) industry. The process provides the capability to pattern circuit boards with small holes without any photoresist collapsing. Hence it becomes a very good material that helps people pattern aluminum and parylene with a very deep trench. The final anchors were released by soaking in DI water. Different anchor shapes can be designed and patterned, depending on the surgical requirement. Results of the fabricated anchors are shown in Figure 5-(f). Parylene tube carrier was made by coating $35 \mu \mathrm{m}$ thick parylene onto glass tubing with $530 \mu \mathrm{m}$ in O.D., which were then cut into desired lengths of $6 \mathrm{~mm}$ with slanted ends on both sides and later free-released in BHF (Figure 4-(c)). Result of fabricated parylene tube carrier is shown in Figure 5-(d).

(a)

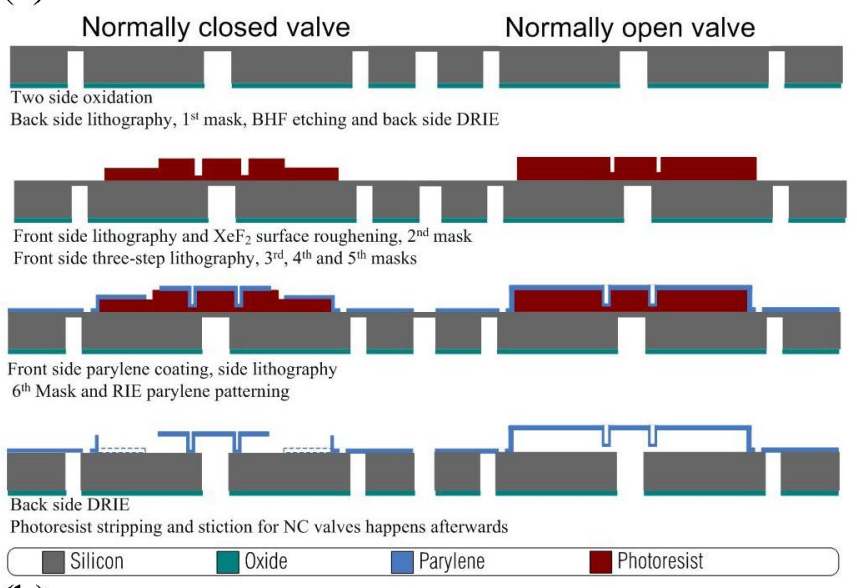

(b)

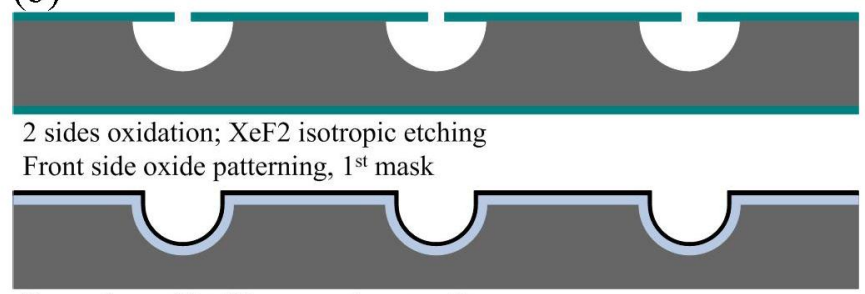

Removing oxide; $20 \mu \mathrm{m}$ parylene coating Al deposition

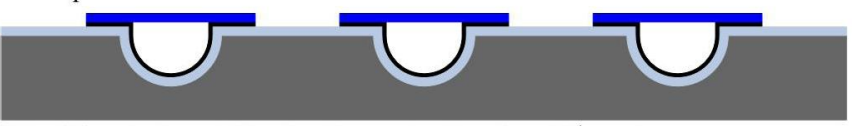

Dry film photoresist laminating and patterning, $2^{\text {nd }}$ mask

Al etching

Parylene RIE etching; Al etching

DI water soaking with ultrasonic parylene releasing

$$
\text { Silicon } \square \text { Parylene } \square \text { Aluminum } \square \text { Dry film photoresist }
$$

(c)

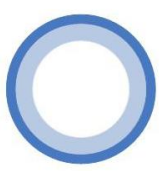

$40 \mu \mathrm{m}$ parylene coating

Two ends slanted by dicing saw BHF glass etching

Parylene
Figure 4: Fabrication procedures of: (a) Microvalves, (b)
Anchors, and (c) Parylene tube carriers.

To package the entire GDD system, one $\mathrm{NC}$ valve and one NO valve were released and then inserted into either ends of the parylene tube carrier. The gap between the microvalves' seats and the tube carrier inner wall was sealed by epoxy (Figure 5-(e)). To mount the finished valved tube onto parylene fixation anchors, tiny epoxy drops was first wiped on the semi sphere recess. Then, the valved tube was assembled onto the recess. Figure 5(g) shows the final assembled GDD with different anchor shapes. Since the O.D. of the parylene tube carrier is 600 $\mu \mathrm{m}(530 \mu \mathrm{m}+70 \mu \mathrm{m}$ parylene coating), it fixes the fixation anchor nicely after assembled. The anchor is so flexible that it can be rolled/folded and insert into the testing Teflon tube thereafter (figure 5-(h)). 

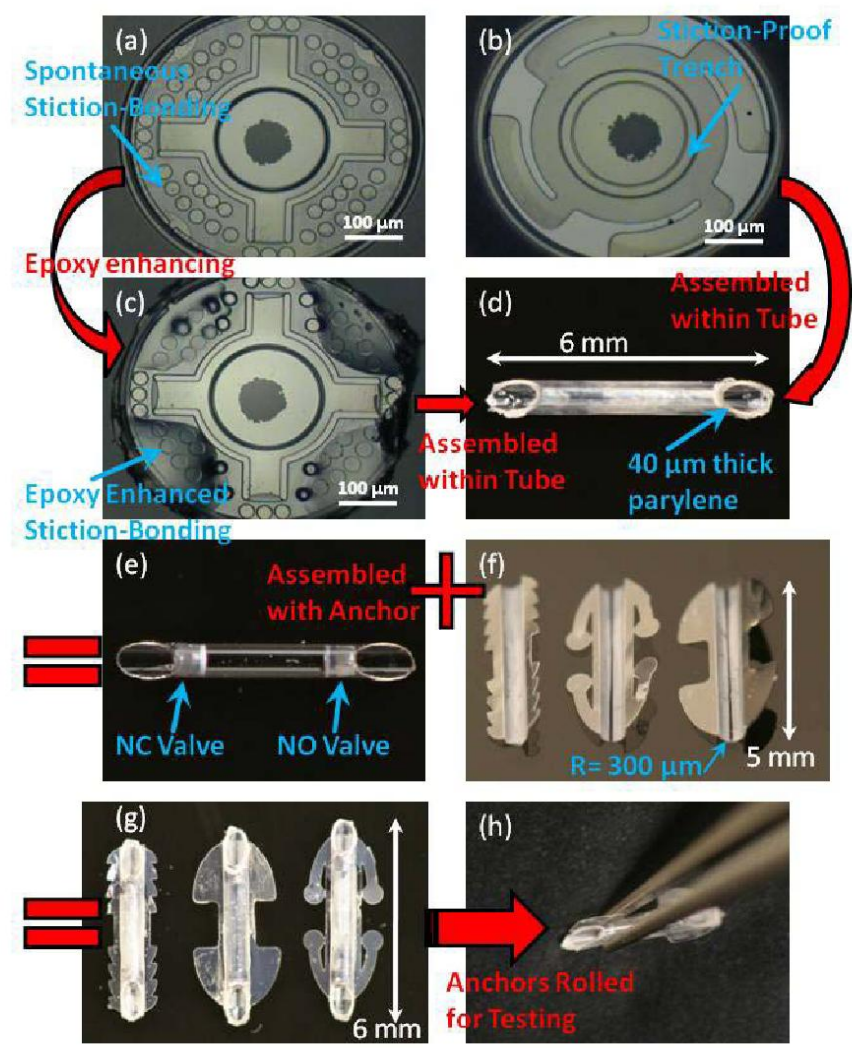

Figure 5: Fabrication results and packaging procedures: Micro graph of (a) NC valve, (b) NO vavle, (c) Epoxy enhanced NC vavle, (d) Hollow parylene tube carrier, (e) One NC and one NO valves sealed in tube carrier (transparent glass tube used here for clarity), (f) Anchors with trenches of $300 \mu \mathrm{m}$ in radius, different anchor shapes designed to facilitate the surgical convenience and future GDD fixation: (left: ragged anchor; middle: foldable anchor; right: rollable squeeze-tail anchor), (g) Complete assembled GDD in top view, (h) Anchors can be rolled folded for testing convenience. Valves are first sealed in the carrier, which is then assembled onto anchors.

\section{BENCH TOP TESTING AND DISCUSSION}

To test the completed GDD, one wingspan was folded by tweezers and inserted into Teflon FET tubing, which has I.D. of $750 \mu \mathrm{m}$. The gap between the GDD and the Teflon tubing was sealed with photoresist and dryied in the air (Figure 6). Water was chosen as the working fluid to mimic the anterior chamber environment. The Teflon tubing was connected to a pressure gauge with resolution up to $0.01 \mathrm{psi}(\sim 5 \mathrm{mmHg})$. The working fluid was pushed by air, which is regulated by a pressure regulator connected between the air source and pressure gauge. Marching speed of the working fluid advancement inside the Teflon tube was measured so that the flow rate can be calculated by knowing the fluid flow speed and the diameter of the Teflon tubing.

Both NC and NO valves were tested separately and the full GDD was then characterized with experimental verification that its behavior meets the standard IOP regulation requirements (Figure 7 ). The single $\mathrm{NC}$ valve starts to pop open at $0.2-0.3 \mathrm{psi}(10-15 \mathrm{mmHg})$, and no obvious flow rate is observed before the cracking pressure. This result meets our simulation expectation and provides the evidence that stiction does provide the required pre-stress force to create the cracking pressure. Fluid in single NO valve flows smoothly before pressure reaches the high limit. The flow rate starts to decrease when the pressure goes up to $0.5 \mathrm{psi}(\sim 25 \mathrm{mmHg})$ and almost closes after 1.0 psi $(-50 \mathrm{mmHg})$. The leak rate is less than $5 \mu \mathrm{L} / \mathrm{min}$. This result proves that the sealing trench designed on NO valve does seal the NO valve opening. The small amount of leak rate may come from the non-flat bottom under the sealing trench which comes from the top surface of the sacrificial photoresist layer. For the dual valve GDD testing, the $\mathrm{NC}$ valve successfully opens at $0.33 \mathrm{psi}(\sim 17 \mathrm{mmHg})$ and $\mathrm{NO}$ valve starts to function at $1.1 \mathrm{psi}(\sim 57 \mathrm{mmHg})$. The reason that off pressure of NO valve in dual valve system is larger than single valve systems is that part of the energy of the flowing fluid is consumed before NO valve by friction and tether deformation of the $\mathrm{NC}$ valve so that only some energy is left to push the NO valve membrane to close the valve. This off pressure delay makes the off pressure of the dual valve system just meet our design requirement to close after $50 \mathrm{mmHg}$. The reported aqueous formation rate is $2-3 \mu \mathrm{L} / \mathrm{min}$ [6], which is satisfied perfectly by our GDD.

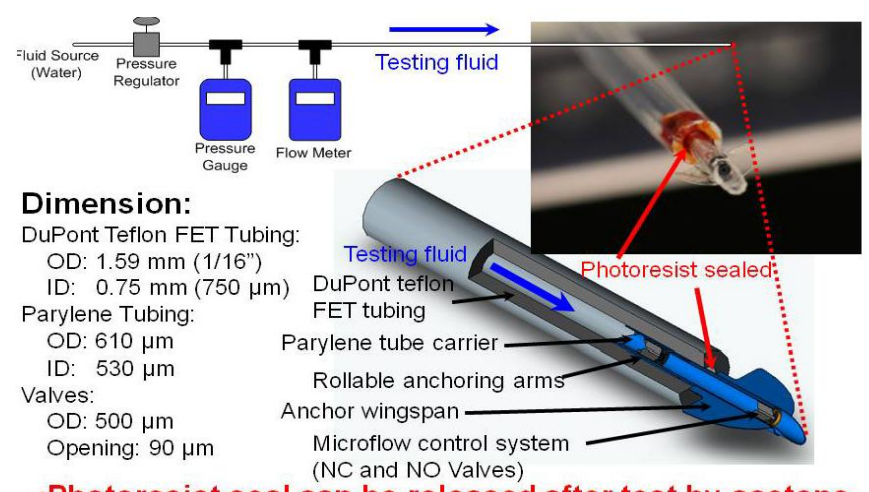

-Photoresist seal can be released after test by acetone. Figure 6: Testing setup of the GDD: Photoresist is painted in the gap between GDD and Teflon tubing for sealing.

Before every GDD is sent to animal surgical testing, the devices are prepared and tested first. After testing is done, the functional GDD is released by soaking into acetone again to ensure functionality of the device. The device wingspan is also stretched back to its original shape after pulling the GDD out of the testing Teflon tubing. The working device is then sent to be sterilized in order to prepare it for later in vivo functionality verification. In vivo animal trial is currently underway to verify the device's bioefficacy in the intraocular environment.

\section{CONCLUSION}

A fully passive, non-power integrated GDD combining micro flow regulation systems, parylene tube carrier and anchors is designed and developed. The parylene anchor with a very deep semi sphere recess is fabricated utilizing the dry film photolithography technique. With this improved surgical features and the newly developed packaging techniques, the overall finished GDD form factor not only still keeps minimally invasive implantation possible, but also prevents dislodging after subconjuctival implantation. 
(a)

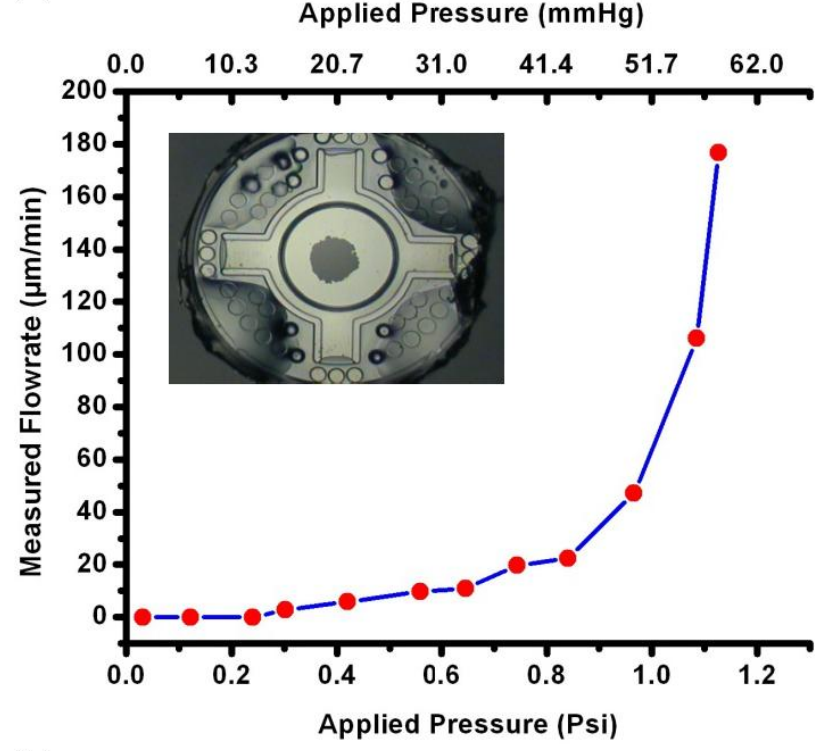

(b)

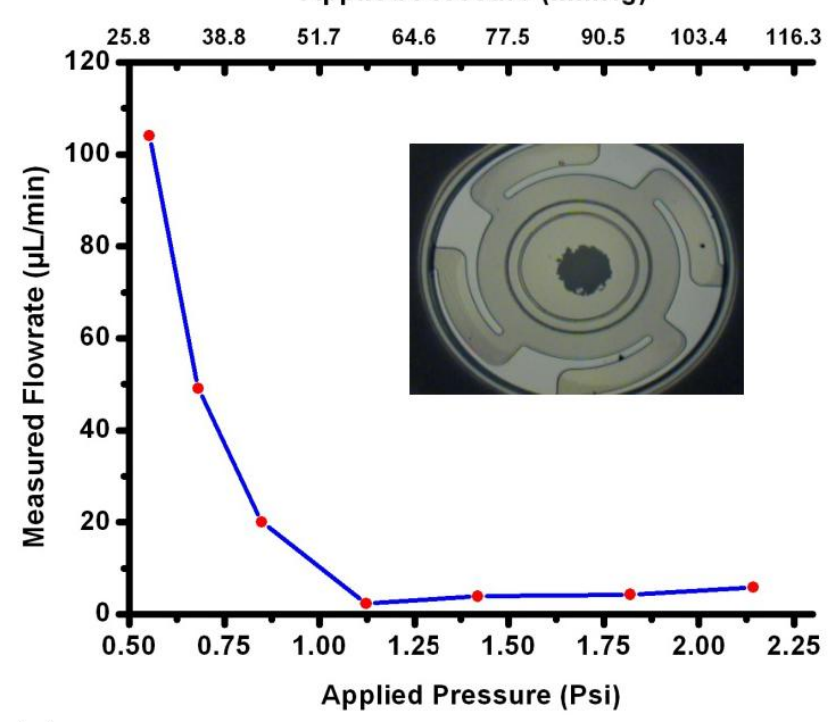

(c)

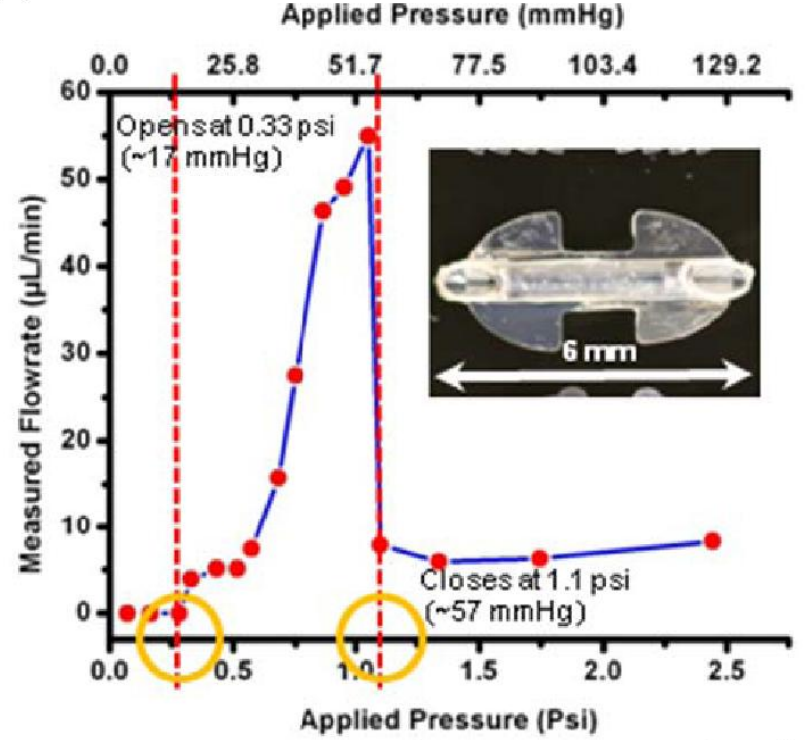

Figure 7: Testing results of (a) Single NC valve, (b) Single NO valve, (c) Dual valved GDD. In (c), the fluid started to flow after $0.33 \mathrm{psi}(\sim 17 \mathrm{mmHg})$, and closed at $1.1 \mathrm{psi}(\sim 57 \mathrm{mmHg})$. Water was chosen as working fluid.
A pressure-bandpass configuration is characterized by on bench verification which illustrates that the results meet medical requirements to treat glaucoma patients. It also shows that the epoxy and photoresist sealing during the packaging and testing procedures secure the system from water leakage very well. In terms of the cracking pressure of the $\mathrm{NC}$ valve, it can be trimmed, depending on the surgical requirements, by changing the number of tethers, length of tethers, parylene thickness, and sacrificial photoresist height.

\section{ACKNOWLEDGEMENTS}

This work was supported by Bausch and Lomb. The authors would also like to thank Mr. Trevor Roper for his valuable fabrication assistance.

\section{REFERENCES}

[1] http://www.ahaf.org/glaucoma/about/glabout.htm

[2] C.-H. Hong, A. Arosemena, D. Zurakowski, R. S. Ayyala, "Glaucoma Drainage Devices: A Systematic Literature Review and Current Controversies", Surv Ophthalmol vol. 50, pp. 48-60, 2005

[3] P.-J. Chen, D. C. Rodger, E. M. Meng, M. S. Humayun, and Y.-C. Tai, "Surface-Micromachined Parylene Dual Valves for On-Chip Unpowered Microflow Regulation," J. Micoelectromech. Syst., vol. 16, pp. 223-231, 2007.

[4] P.-J. Chen, D. C. Rodger, S. Saati, J. C. Altamirano, C.-H. Lin, R. Agrawal, R. Varma, M. S. Humayun, and Y.-C. Tai, "Implementation of Microfabricated Sutureless Flexible Parylene Tissue Anchors on Minimally Invasive Biomedical Implants", in Proc. MicroTAS 2007, Paris, France, pp. 518-520.

[5] J. C.-H. Lin, P.-J. Chen, S. Saati, R. Varma, M. Humayun, Y.-C. Tai, "Implantable MicrovalvePackaged Glaucoma Drainage Tube", in Tech. Digest 13 th Solid State Sens., Actuators, and Microsyst. Workshop, Hilton Head, SC, Jun. 1-5, 2008, pp. 146149.

[6] http://www.nyee.edu/pdf/solomonaqhumor.pdf

[7] N. Tas, T. Sonnenberg, H. Jansen, R. Legtenberg, and M. Elwenspoek, "Stiction in Surface Micromachining," J. Micromech. Microeng., vol. 6, pp. 385-397, 1996.

[8] H. Zhang, S. Wang, and Y. Xu, "Study and Applications of A Parylene Self-Sealing Structure," Technical Digest, in Tech. Digest 19th IEEE International Conference on MicroElectroMechanical Systems (MEMS 2006), Istanbul, Turkey, Jan. 22-26, pp. 282-285.

[9] C. T. Leondes, MEMS/NEMS Handbook Technigues and Applications Volume 5, Springer Science+Business Media, Inc., New York, 2006.

[10]K. Dietz, Dry Film Photoresist Processing Technology, Electrochemical Publications Ltd, Port Erin, British Isles, 2001. 